Research Article

\title{
Optimal Correction of Adult Spinal Deformities Requires Restoration of Distal Lumbar Lordosis
}

\author{
S. Pesenti, S. Prost, A. Muñoz McCausland, K. Farah, P. Tropiano, S. Fuentes, \\ and B. Blondel \\ Aix-Marseille Université, APHM, CNRS, ISM, Hôpital de La Timone, Spine Unit 264 Rue Saint Pierre, Marseille 13005, France \\ Correspondence should be addressed to B. Blondel; benjamin.blondel@ap-hm.fr
}

Received 15 February 2021; Revised 20 April 2021; Accepted 1 May 2021; Published 7 May 2021

Academic Editor: Hiroshi Hashizume

Copyright (C) 2021 S. Pesenti et al. This is an open access article distributed under the Creative Commons Attribution License, which permits unrestricted use, distribution, and reproduction in any medium, provided the original work is properly cited.

\begin{abstract}
Purpose. The aim of this study is to analyze results according to postoperative pelvic incidence-lumbar lordosis (PI-LL) mismatch in the management of adult spine deformity (ASD) patients. Recently, it has been reported that in addition to lumbar lordosis amount, lordosis repartition between its proximal and distal parts was crucial. Methods. We enrolled 77 consecutive ASD patients who underwent posterior spinal fusion and deformity correction between 2015 and 2018. On preoperative and 1-year follow-up radiographs, we analyzed different parameters such as L1-S1 lumbar lordosis, L1-L4 proximal lordosis (PLL), L4-S1 distal lordosis (DLL), pelvic tilt (PT), sagittal vertical axis (SVA), and PI-LL mismatch. Comparisons were performed according to postoperative PI-LL mismatch (defined as "aligned" when PI-LL was $<10^{\circ}$ ). The relationship between lordosis distribution and postoperative alignment status was investigated. Results. On the whole series, average lumbar lordosis, SVA, and PI-LL improved $\left(28.2^{\circ}\right.$ vs.43.5 $5^{\circ}$ 82 vs. $51 \mathrm{~mm}$, and $26^{\circ}$ vs. $14^{\circ}$, all $p<0.001$, respectively). On the other hand, PT remained unchanged $\left(30^{\circ}\right.$ vs. $\left.28^{\circ}, p>0.05\right) .35$ patients were classified as "aligned" and 42 as "not aligned." Patients from the "aligned" group had a significantly lower PI than patients from the "not aligned" group $\left(52^{\circ}\right.$ vs. $\left.61^{\circ}, p=0.009\right)$. Postoperative PLL was not different between groups $\left(18^{\circ}\right.$ vs. $16^{\circ}$ $p>0.05)$, whereas DLL was significantly higher in the "aligned" group ( $31^{\circ}$ vs. $\left.22^{\circ}, p=0.003\right)$. PI-LL was significantly correlated to DLL (rho $=0.407, p<0.001$ ) but not with PLL ( $r h o=0.110, p=0.342)$. Conclusions. Our results revealed that in ASD patients, postoperative malalignment was associated with a lack of DLL restoration. "Not aligned" patients had also a significantly higher pelvic incidence. Specific attention must be paid to restore optimal distal lumbar lordosis in order to set the amount and the distribution of optimal postoperative lumbar lordosis.
\end{abstract}

\section{Introduction}

Adult spinal deformity covers a broad range of pathologies and can be responsible for disability and altered quality of life [1]. When a surgical procedure is indicated, the aim is to restore sagittal and coronal alignment. Since the last decade, it has been largely reported that correction of sagittal alignment was of primary importance and associated with better outcomes [2-4].

ASD is commonly described with the SRS-Schwab classification [5] that also serves as guideline for sagittal correction $\left(\mathrm{SVA}<50 \mathrm{~mm}, \mathrm{PI}-\mathrm{LL}<10^{\circ}\right.$, and $\left.\mathrm{PT}<20^{\circ}\right)$.
However, despite recent advances such as age-related spinopelvic alignment thresholds [6], postoperative sagittal objectives are reached in less than $50 \%$ of the cases [7].

The key parameter to evaluate postoperative alignment is the PI-LL mismatch. This parameter has been reported as a predictor of success and is correlated with health-related quality of life scores [8]. However, taking into account only the L1-S1 lumbar lordosis (LL) can be misleading for two reasons [8]:

The L4-S1 DLL was reported to be almost constant Pelvic incidence is significantly related to the L1-L4 PLL 
It seems therefore crucial to evaluate not only the global amount of LL restoration but also the PI-LL mismatch according to PLL and DLL.

This study aimed to analyze the results of ASD surgical management according to postoperative PI-LL mismatch with the hypothesis that aligned patients had a better restoration of the DLL.

\section{Methods}

2.1. Study Design and Patient Population. After institutional review board approval (IRB 00009118), we conducted a retrospective analysis of a prospectively maintained database of ASD patients operated between 2015 and 2018. Prior to inclusion, every patient signed an informed consent.

Inclusion criteria were as follows: all adult patients managed surgically for ASD with a posterior fixation that included all the lumbar spine (upper level instrumented vertebra L1 or above, lower instrumented vertebra S1 or below), primary surgery or revision case, pre and postoperative full spine $\mathrm{X}$-rays available, and one-year minimal follow-up (including full spine X-rays).

For each patient, demographic data, clinical scores (lumbar VAS and Oswestry Disability Index), and full spine $\mathrm{X}$-rays (AP and lateral) were obtained preoperatively, in the immediate postoperative period, and at one-year of followup. Complications during the follow-up period were systematically noted.

The surgical procedure consisted of a posterior fusion and bone resection if required (Grade 2 or 3 of the Schwab classification [9]).

2.2. Radiographic Parameters. On lateral radiographs, the following parameters were measured: T4-T12 thoracic kyphosis (TK), L1-S1 lumbar lordosis, L1-L4 PLL, L4-S1 DLL, SVA, pelvic parameters (PI and PT), and mismatch between pelvic incidence and lumbar lordosis (PI-LL).

2.3. Statistical Analysis. Data were formulated as means and standard deviations. Comparisons were carried out using Student's t-test for normally distributed variables. The population was analyzed as a whole and then stratified according to the 1-year postoperative PI-LL mismatch. Patients were classified as the "aligned" group when postoperative PI-LL was below $10^{\circ}$ and "not aligned" otherwise. The two groups were compared with regards to radiographic parameters and mechanical complications. The relationship between PI-LL mismatch and lumbar lordosis distribution was investigated using Spearman correlation tests. The significance level was set at 95\% (i.e., $p<0.05$ ).

\section{Results}

3.1. Population and Surgical Data. Among the 97 patients who underwent ASD surgical correction in our institution during the inclusion period, 77 patients met inclusion criteria for the present study. Fifty-six women and 21 men with a mean age of 66.5 years old $(S D=8.8)$ were included. On average, 14 levels were fused during the procedure $(\mathrm{SD}=3)$, and lower instrumented level included iliac screws for 43 patients and S1 screws for 34 patients.

3.2. Global Radiographic Analysis. On the whole series, the mean preoperative $\mathrm{PI}$ was $54.3^{\circ} \mathrm{SD}=13$ and did not change during follow-up.

With regards to regional parameters, a significant improvement of LL and TK was noted between preoperative and last follow-up evaluation $\left(28.2^{\circ}\right.$ vs. $43.5^{\circ}, p<0.001$ and $30.2^{\circ}$ vs. $43.9^{\circ}, p<0.001$, respectively).

SVA and PI-LL mismatch were significantly reduced (81.9 mm vs. $50.9 \mathrm{~mm}, p<0.001$ and $26^{\circ}$ vs. $13.5^{\circ}, p<0.001$, respectively). On the other hand, PT remained unchanged between pre and 1-year assessment $\left(29.9^{\circ}\right.$ vs. $\left.28.1^{\circ}, p>0.05\right)$.

3.3. Aligned vs. Nonaligned Group Analysis. Thirty-five patients were classified as "aligned" (PI-LL $<10^{\circ}$ ) and 42 were classified as "not aligned" (PI-LL $>10^{\circ}$ ). Of the 42 "not aligned" patients, 17 were revision cases, 10 requiring a grade 3 osteotomy ( 8 in L3 and 2 in L4). Among the 35 "aligned" patients, 19 were revision cases, 7 requiring a grade 3 osteotomy (5 in L3 and 2 in L4) (Figures 1 and 2).

Preoperatively, "not aligned" patients had a significantly higher pelvic incidence, higher pelvic tilt, smaller lumbar lordosis, and thoracic kyphosis, despite a nonsignificantly different sagittal vertical axis (Table 1).

With regards to preoperative LL distribution, the L1-L4 PLL $\left(1.5^{\circ}\right.$ vs. $\left.08^{\circ}, p=0.9\right)$ and the L4-S1 DLL (33.3 vs. $28.3^{\circ}$, $p=0.254$ ) were not significantly different between groups (Table 1).

Postoperatively, "aligned" patients showed a significantly higher improvement of LL, TK, and SVA (Table 2).

With regards to postoperative LL distribution, the L1-L4 PLL was not significantly different between groups $\left(18^{\circ}\right.$ vs. $\left.16^{\circ} p>0.05\right)$, but the L4-S1 DLL was significantly higher in the "aligned" group ( $31^{\circ}$ vs. $\left.22^{\circ}, p=0.003\right)$.

The PI-LL mismatch was significantly correlated with the L4-S1 DLL (rho $=0.407, p<0.001)$ but not with the L1-L4 PLL (rho $=0.110, p=0.342$ ). This significant correlation revealed that restoration of the PI-LL mismatch was significantly related to the correction of the distal lumbar lordosis.

\subsection{Postoperative Complications and Clinical Evaluation.} During the follow-up period, 7 postoperative infections were noted (4 in the "not aligned" group and 3 in the "aligned" group) that required surgical debridement and adapted antibiotics.

At last follow-up, 1 patient out of $35(3 \%)$ in the aligned group had a mechanical complication (rod breakage) that required revision surgery. Six patients out of 42 (14\%) of the "not aligned" group had a mechanical complication that required revision surgery (4 rod breakage and 2 proximal junctional failure).

With regards to final follow-up clinical scores, patients from the "aligned" group had a significantly lower lumbar 


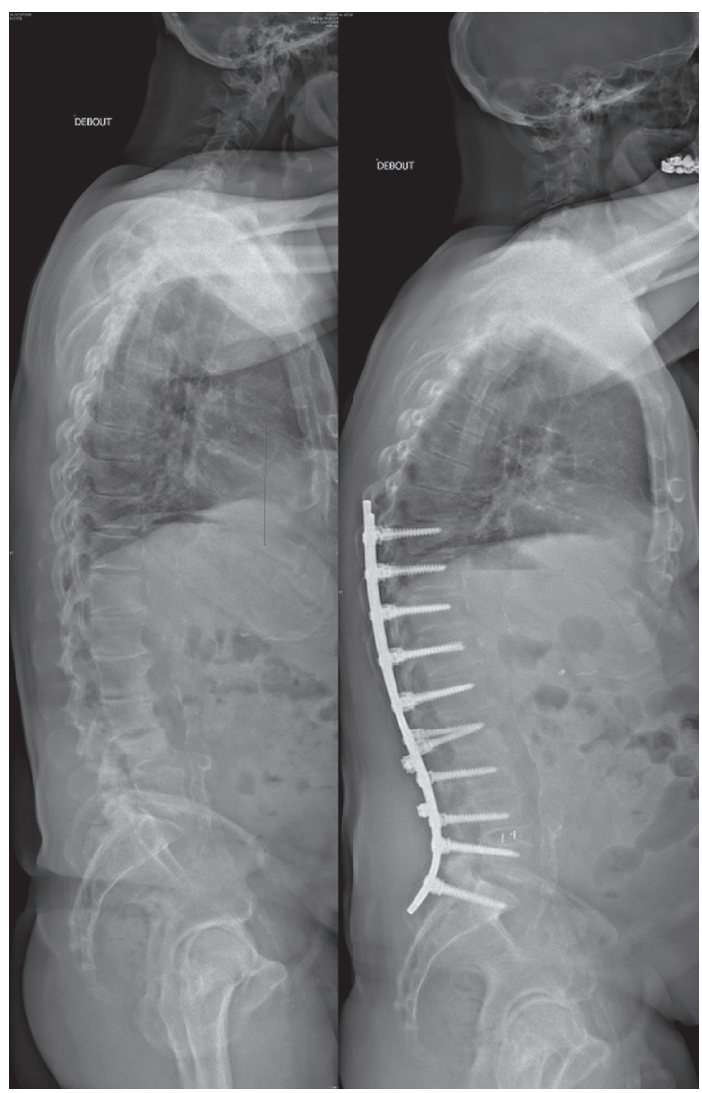

FIgURE 1: Clinical example of a patient from the "aligned group" with a low PI. Primary case, T9-S1 posterior fixation, multiples grade 2 osteotomies. Preoperative measurements (left) were $\mathrm{PI}=41^{\circ}, \mathrm{PT}=21^{\circ}, \mathrm{PI}-\mathrm{LL}=21, \mathrm{LL}=-20^{\circ}, \mathrm{TK}=22^{\circ}$, and $\mathrm{SVA}=42 \mathrm{~mm}$. One-year measurements (right) were PT $=17^{\circ}, \mathrm{PI}-\mathrm{LL}=0, \mathrm{LL}=-41^{\circ}, \mathrm{PLL}=-8^{\circ}, \mathrm{DLL}=-33^{\circ}, \mathrm{TK}=41^{\circ}$, and $\mathrm{SVA}=41 \mathrm{~mm}$.

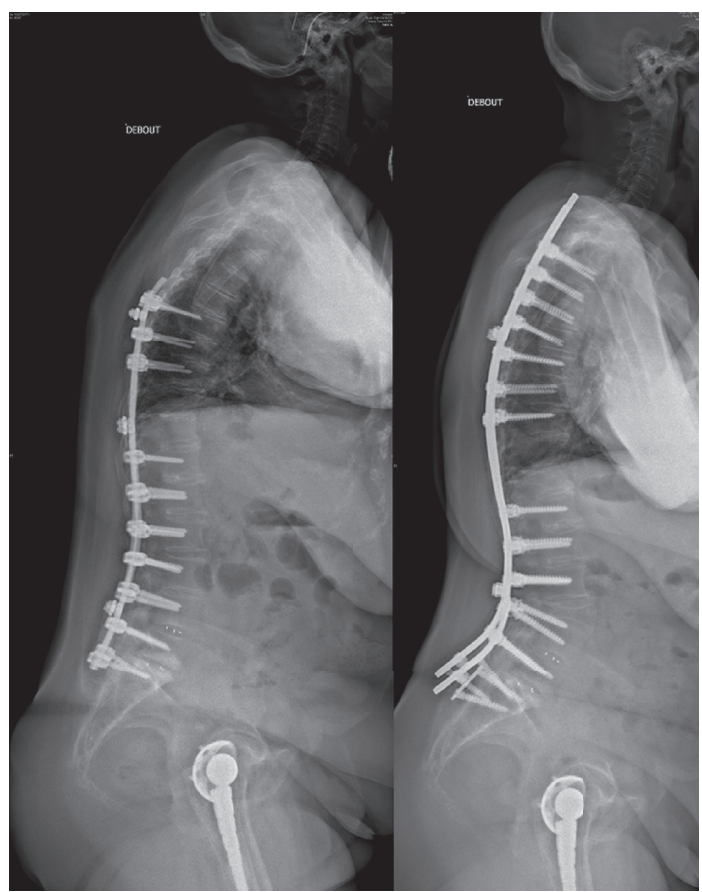

FIgURE 2: Clinical example of a patient form the "not aligned" group with a high PI. Revision case, T3-S2 posterior fixation, L3 grade 3 osteotomy. Preoperative measurements (left) were $\mathrm{PI}=59^{\circ}, \mathrm{PT}=34^{\circ}, \mathrm{PI}-\mathrm{LL}=32^{\circ}, \mathrm{LL}=-27^{\circ}$, TK $=3^{\circ}$, and SVA $=93 \mathrm{~mm}$. One-year measurements (right) were PT $=25^{\circ}, \mathrm{PI}-\mathrm{LL}=15^{\circ}, \mathrm{LL}=-44^{\circ}, \mathrm{PLL}=-29^{\circ}, \mathrm{DLL}=-15^{\circ}, \mathrm{TK}=36^{\circ}$, and SVA $=91 \mathrm{~mm}$. 
TABLE 1: Preoperative values of radiographic parameters between "aligned" and "not aligned" groups.

\begin{tabular}{lccc}
\hline Preoperative & Aligned & Not aligned & $p$ value \\
\hline Pelvic incidence $\left({ }^{\circ}\right)$ & 52 & 61 & 0.009 \\
Pelvic tilt $\left({ }^{\circ}\right)$ & 26 & 33 & 0.001 \\
L1-S1 lumbar lordosis $\left({ }^{\circ}\right)$ & 34 & 23 & 0.004 \\
L1-L4 PLL $\left({ }^{\circ}\right)$ & 1.5 & 0.8 & 0.9 \\
L4-S1 DLL $\left({ }^{\circ}\right)$ & 33.3 & 28.3 & 0.254 \\
PI-LL $\left({ }^{\circ}\right)$ & 16 & 34 & $<0.001$ \\
T4-T12 thoracic kyphosis $\left({ }^{\circ}\right)$ & 37 & 25 & 0.008 \\
Sagittal vertical axis $(\mathrm{mm})$ & 71 & 91 & $>0.05$ \\
\hline
\end{tabular}

TABle 2: Postoperative values of radiographic parameters between "aligned" and "not aligned" groups.

\begin{tabular}{lccc}
\hline Postoperative & Aligned & Not aligned & $p$ value \\
\hline Pelvic incidence $\left({ }^{\circ}\right)$ & 52 & 61 & 0.009 \\
Pelvic tilt $\left({ }^{\circ}\right)$ & 24 & 33 & $<0.001$ \\
L1-S1 lumbar lordosis $\left({ }^{\circ}\right)$ & 49 & 37 & $<0.001$ \\
L1-L4 PLL $\left({ }^{\circ}\right)$ & 18 & 16 & 0.39 \\
L4-S1 DLL $\left(^{\circ}\right)$ & 31 & 22 & 0.003 \\
PI-LL $\left({ }^{\circ}\right)$ & 4 & 23 & $<0.001$ \\
T4-T12 thoracic kyphosis $\left({ }^{\circ}\right)$ & 48 & 40 & 0.002 \\
Sagittal vertical axis $(\mathrm{mm})$ & 23 & 63 & $<0.001$ \\
\hline
\end{tabular}

VAS (2.8/10 vs. $4.8 / 10, p=0.028)$. ODI scores were not significantly different between groups (“aligned” $29 \%$ vs. "not aligned" 37\%, $p=0.095)$.

\section{Discussion}

Surgical management of ASD remains challenging, and optimal management is still under debate. The complication rate after realignment procedures has been reported around $16.5 \%$ at 2 years of follow-up and $50 \%$ at 10 years of followup $[10,11]$.

According to the literature, a large proportion (up to $50 \%$ ) of patients remains undercorrected after surgery [7]. Perfect understanding of this undercorrection is difficult especially, as alignment can still change postoperatively. According to McDowell et al. [12], a significant improvement of SVA can be seen during the first postoperative year without further modifications. However, this progressive correction of the SVA was also associated with an increase of pelvic tilt which can be considered as a compensatory mechanism in undercorrected patients.

Restoration of pelvic tilt might therefore be a crucial point for sagittal realignment procedures, especially in patients with a high pelvic incidence. Lafage et al. [13] suggested that patients with a high preoperative pelvic retroversion (related to a high pelvic incidence) require larger lumbar osteotomy procedures. In our series, pelvic tilt was the most difficult parameter to restore, with a nonsignificant postoperative correction. This lack of correction can be related to an insufficient bone resection, but it can also be associated with the level of the osteotomy. Mainly, L3 grade 3 osteotomies have been performed in our experience, and in order to achieve a proper spinopelvic alignment, performing osteotomy at a lower lumbar level (L4 or L5) can increase pelvic tilt correction with an average of $2^{\circ}$ per level $[14,15]$.
Another important parameter for realignment procedures is the postoperative location of the LL apex. Based on Roussouly classification, Pizones et al. recently reported a decrease in mechanical complications with an adapted postoperative lumbar apex position and lumbar shape restoration $[16,17]$. Of note, besides sagittal alignment goals, the use of multiple rods constructs has also been reported as a safe and effective method to reduce implant failures $[18,19]$, but this strategy was not used in our experience.

In our study, the choice was made to stratify patients according to postoperative PI-LL mismatch. Our results revealed that postoperatively "aligned" patients had a preservation of L4-S1 DLL and a higher pelvic incidence when compared to "not aligned" patients. These results are consistent with the study of Ylgor et al. who suggested taking into account lumbar lordosis distribution [20]. While L4-S1 DLL represents around $2 / 3$ of global lordosis, it has been reported that the amount of this L4-S1 DLL was constant and independent from pelvic incidence. However, pelvic incidence is significantly correlated to the L1-L4 PLL [8]. As a whole, these results suggest that the loss of lumbar lordosis mainly occurs in the L4-S1 DLL. As a consequence, failure to restore DLL is correlated with an increased risk of proximal junctional failure [21], and DLL should be preserved or restored around $36^{\circ}$ [8].

Results of this study confirm that restoration of L4-S1 DLL is a crucial objective for ASD patients as recently reported by Lafage et al. [22] as $1^{\circ}$ of L4-S1 DLL correction produces $10 \mathrm{~mm}$ change in SVA and $0.5^{\circ}$ in PT. Two strategies can therefore be advocated to reach this goal. First option is based on an anterior approach with intersomatic lordotic cages prior to the posterior fixation. According to recent studies [23-25], this technique can eliminate the need for a grade 3 osteotomy during the posterior approach, especially in primary cases. The second option is to perform L4 or even L5 grade 3 osteotomy [11]. 
This study presents several limitations such as the oneyear follow-up and a limited number of patients included. Further studies will be needed to confirm these results.

\section{Conclusion}

Adult spinal deformity is a frequent and challenging condition for spine physicians. Based on postoperative PI-LL mismatch, the results of this study revealed that maintenance or restoration of L4-S1 DLL is crucial for postoperative alignment. Specific attention must be paid to restore optimal distal lumbar lordosis in order to decrease the rate of undercorrected patients and to improve outcomes.

\section{Data Availability}

The data used to support the findings of this study are available from the corresponding author upon request.

\section{Ethical Approval}

The study was approved by IRB 00009118.

\section{Consent}

Each patient signed an informed consent prior to inclusion.

\section{Conflicts of Interest}

B Blondel is a consultant at Medicrea International, Implanet, $3 \mathrm{M}$, Vexim-Stryker. S Fuentes is a consultant at Medicrea International, Medtronic, Stryker. P Tropiano is a consultant at France Rachis, Depuy Synthes, LDR Zimmer. S Prost, S Pesenti, A. Muñoz McCausland, and K Farah declare that they have no conflicts of interest.

\section{References}

[1] F. J. Schwab, B. Blondel, S. Bess et al., "Radiographical spinopelvic parameters and disability in the setting of adult spinal deformity," Spine, vol. 38, no. 13, pp. E803-E812, 2013.

[2] B. Blondel, F. Schwab, B. Ungar et al., "Impact of magnitude and percentage of global sagittal plane correction on healthrelated quality of life at 2-years follow-up," Neurosurgery, vol. 71, no. 2, pp. 341-348, 2012, discussion 348.

[3] J. S. Smith, C. I. Shaffrey, V. Lafage et al., "Comparison of best versus worst clinical outcomes for adult spinal deformity surgery: a retrospective review of a prospectively collected, multicenter database with 2-year follow-up," Journal of Neurosurgery: Spine, vol. 23, no. 3, pp. 349-359, 2015.

[4] J. Simon, P.-M. Longis, and N. Passuti, "Correlation between radiographic parameters and functional scores in degenerative lumbar and thoracolumbar scoliosis," Orthopaedics \& Traumatology: Surgery \& Research, vol. 103, no. 2, pp. 285290, 2017.

[5] F. Schwab, B. Ungar, B. Blondel et al., "Scoliosis research society-schwab adult spinal deformity classification," Spine, vol. 37, no. 12, pp. 1077-1082, 2012.

[6] R. Lafage, F. Schwab, V. Challier et al., "Defining spino-pelvic alignment thresholds," Spine, vol. 41, no. 1, pp. 62-68, 2016.

[7] B. Moal, F. Schwab, C. P. Ames et al., "Radiographic outcomes of adult spinal deformity correction: a critical analysis of variability and failures across deformity patterns," Spine Deformity, vol. 2, no. 3, pp. 219-225, 2014.

[8] S. Pesenti, R. Lafage, D. Stein et al., "The amount of proximal lumbar lordosis is related to pelvic incidence," Clinical Orthopaedics \& Related Research, vol. 476, no. 8, pp. 1603-1611, 2018.

[9] F. Schwab, B. Blondel, E. Chay et al., "The comprehensive anatomical spinal osteotomy classification," Neurosurgery, vol. 74, no. 1, pp. 112-120, 2014, discussion 120.

[10] P. G. Passias, A. Soroceanu, S. Yang et al., "Predictors of revision surgical procedure excluding wound complications in adult spinal deformity and impact on patient-reported outcomes and satisfaction," Journal of Bone and Joint Surgery, vol. 98, no. 7, pp. 536-543, 2016.

[11] A. Sebaaly, M. Gehrchen, C. Silvestre et al., "Mechanical complications in adult spinal deformity and the effect of restoring the spinal shapes according to the Roussouly classification: a multicentric study," European Spine Journal, vol. 29, no. 4, pp. 904-913, 2020.

[12] M. M. McDowell, Z. J. Tempel, G. S. Gandhoke et al., "Evolution of sagittal imbalance following corrective surgery for sagittal plane deformity," Neurosurgery, vol. 81, no. 1, pp. 129-134, 2017.

[13] V. Lafage, B. Blondel, J. S. Smith et al., "Preoperative planning for pedicle subtraction osteotomy: does pelvic tilt matter?" Spine Deformity, vol. 2, no. 5, pp. 358-366, 2014.

[14] V. Lafage, F. Schwab, S. Vira et al., "Does vertebral level of pedicle subtraction osteotomy correlate with degree of spinopelvic parameter correction?" Journal of Neurosurgery: Spine, vol. 14, no. 2, pp. 184-191, 2011.

[15] E. Ferrero, B. Liabaud, J. K. Henry et al., "Sagittal alignment and complications following lumbar 3-column osteotomy: does the level of resection matter?" Journal of Neurosurgery: Spine, vol. 27, no. 5, pp. 560-569, 2017.

[16] J. Pizones, L. Moreno-Manzanaro, L. Moreno-Manzanaro et al., "Restoring the ideal Roussouly sagittal profile in adult scoliosis surgery decreases the risk of mechanical complications," European Spine Journal, vol. 29, no. 1, pp. 54-62, 2020.

[17] J. Pizones, F. J. S. Perez-Grueso, F. J. S. Perez-Grueso et al., "Ideal sagittal profile restoration and ideal lumbar apex positioning play an important role in postoperative mechanical complications after a lumbar PSO," Spine Deformity, vol. 8, no. 3, pp. 491-498, 2020.

[18] F. Guevara-Villazón, L. Boissiere, K. Hayashi et al., "Multiplerod constructs in adult spinal deformity surgery for pelvicfixated long instrumentations: an integral matched cohort analysis," European Spine Journal, vol. 29, no. 4, pp. 886-895, 2020.

[19] S.-J. Hyun, L. G. Lenke, Y.-C. Kim, L. A. Koester, and K. M. Blanke, "Comparison of standard 2-rod constructs to multiple-rod constructs for fixation across 3-column spinal osteotomies," Spine, vol. 39, no. 22, pp. 1899-1904, 2014.

[20] C. Yilgor, N. Sogunmez, Y. Yavuz et al., "Relative lumbar lordosis and lordosis distribution index: individualized pelvic incidence-based proportional parameters that quantify lumbar lordosis more precisely than the concept of pelvic incidence minus lumbar lordosis," Neurosurgical Focus, vol. 43, no. 6, p. E5, 2017.

[21] R. Lafage, I. Obeid, B. Liabaud et al., "Location of correction within the lumbar spine impacts acute adjacent-segment kyphosis," Journal of Neurosurgery: Spine, vol. 30, no. 1, pp. 69-77, 2019.

[22] R. Lafage, F. Schwab, J. Elysee et al., "Surgical planning for adult spinal deformity: anticipated sagittal alignment 
corrections according to the surgical level," Global Spine Journal, vol. 11, Article ID 219256822098850, 2021.

[23] P. Hosseini, G. M. Mundis, R. K. Eastlack et al., "Preliminary results of anterior lumbar interbody fusion, anterior column realignment for the treatment of sagittal malalignment," Neurosurgical Focus, vol. 43, no. 6, p. E6, 2017.

[24] J.-C. Leveque, V. Yanamadala, Q. D. Buchlak, and R. K. Sethi, "Correction of severe spinopelvic mismatch: decreased blood loss with lateral hyperlordotic interbody grafts as compared with pedicle subtraction osteotomy," Neurosurgical Focus, vol. 43, no. 2, p. E15, 2017.

[25] A. A. Theologis, G. M. Mundis, S. Nguyen et al., "Utility of multilevel lateral interbody fusion of the thoracolumbar coronal curve apex in adult deformity surgery in combination with open posterior instrumentation and L5-S1 interbody fusion: a case-matched evaluation of 32 patients," Journal of Neurosurgery: Spine, vol. 26, no. 2, pp. 208-219, 2017. 